\title{
IMPLICIT FUNCTIONS OF ALMOST PERIODIC FUNCTIONS*
}

\author{
BY R. H. CAMERON $\dagger$
}

1. Introduction. It is a well known fact that a uniformly continuous single-valued function of a uniformly almost periodic (u.a.p.) function is u.a.p., and that its module is contained in the module of the original function. However, the same statements cannot be made concerning multiple-valued functions; for instance, a u.a.p. function can be constructed which is never zero and whose two continuous square root functions are neither of them u.a.p. (or even Stepanoff a.p.). Moreover, when a square root (or other multiple-valued) function is a.p., it need not have a module contained in the module of the original. This is illustrated by $e^{i x}$ whose module consists of all integers, but whose square roots $e^{i x / 2}$ and $-e^{i x / 2}$ have modules consisting of all integers and half integers.

It is the aim of this paper to obtain sufficient conditions under which multiple-valued functions (implicit functions) of an a.p. function are a.p., and to investigate the module of such solutions. In the main, u.a.p. functions will be considered, but some results will also be obtained for S.a.p. functions.

2. Statement of Theorem 1. In the following theorem, $x$ denotes the real variable while $f(x), z$, and $F(x, z)$ are either all real or all complex.

THEOREM 1. Let $f(x)$ be a continuous bounded function defined for all values of $x$; let 5 be the set of values which $f(x)$ can assume and let $\Im$ be the set of ordered pairs $(x, z)$ such that $|f(x)-z|<\mu$, $\mu>0$. Let $F(x, z)$ be a function having the following properties:

(a) $F(x, z)$ is u.a.p. in $x$ uniformly in z for all z in (5.

(b) $F(x, z)$ is uniformly continuous in z uniformly with respect to $x$ for all $(x, z)$ on $\mathfrak{S}$.

(c) $\partial F / \partial z=F_{z}(x, z)$ exists everywhere on $\mathfrak{\$}$.

(d) $F[x, f(x)]=0$ and $\left|F_{z}[x, f(x)]\right|>\lambda>0$ for all $x$.

* Presented to the Society, October 28, 1933, and December 1, 1933.

$\dagger$ National Research Fellow. 
(e) $F_{z}(x, z)$ is continuous in $z$ at $z=f(x)$ uniformly for all $x$. Finally, let $R$ be the set of sequences of real numbers $h_{1}, h_{2}, \cdots$ such that $\lim _{k \rightarrow \infty} f\left(h_{k}\right)$ exists and $\lim _{k \rightarrow \infty} F\left(x+h_{k}, z\right)=F(x, z)$ uniformly for all $x$ and all $z$ in $\mathbb{E}$.

Then it follows that

(a) $f(x)$ is u.a.p.

(b) The number $P$ of numbers which can be approached by sequences of $\mathcal{R}$ is finite.

(c) The module of $f(x)$ is contained in the set of numbers obtained by dividing by $P$ each element of the common module of $F(x, z)$ for zin (5.

3. Proof. Because of the hypotheses on $F(x, z)$, we have a uniform implicit function theorem as follows. There exist positive numbers $\eta$ and $\nu$ such that to each pair $(x, w)$ of which $w$ is a complex number satisfying $|w| \leqq \nu$ there corresponds one and only one complex number $Z_{x}(w)$ which satisfies simultaneously

$$
\left|Z_{x}(w)-f(x)\right| \leqq \eta \text { and } F\left[x, Z_{x}(w)\right]=w .
$$

Moreover $Z_{x}(w)$ is uniformly continuous in $w$ uniformly with respect to $x$ for $|w| \leqq \nu$ and all $x$; and of course $Z_{x}(0)=f(x)$.

It will be shown in this section that if $h_{1}, h_{2}, \cdots$ belong to $R$, then $\lim _{k \rightarrow \infty} f\left(x+h_{k}\right)$ exists uniformly in $x$ for all $x$. Let $h_{1}, h_{2}, \cdots$ be any sequence of $R$, and let $\epsilon>0$. Then corresponding to $\epsilon_{1}$, the smaller of $\epsilon$ and $\eta / 2$, choose a positive number $\delta \leqq \nu$ such that whenever $|w| \leqq \delta$, then, for all $x$,

$$
\left|Z_{x}(w)-f(x)\right| \leqq \epsilon_{1} \text {. }
$$

Let $N$ be so great that $\left|f\left(h_{m}\right)-f\left(h_{n}\right)\right| \leqq \epsilon_{1}$ when $m>N$ and $n>N$, and that, for all $x$ and all $z$ in $\mathfrak{C}$,

$$
\left|F\left(x+h_{m}, z\right)-F\left(x+h_{n}, z\right)\right| \leqq \delta .
$$

Now suppose that there exists for some $m>N$ and $n>N$ a real number $x_{0}$ such that

$$
\eta>\left|f\left(x_{0}+h_{m}\right)-f\left(x_{0}+h_{n}\right)\right|>\epsilon_{1} ;
$$

and let

$$
w_{0}=F\left[x_{0}+h_{n}, f\left(x_{0}+h_{m}\right)\right] .
$$


Then, since $F\left[x_{0}+h_{m}, f\left(x_{0}+h_{m}\right)\right]=0$, we have from (3) that $\left|w_{0}\right| \leqq \delta \leqq \nu$. It follows from the uniqueness of the function $Z_{x}(w)$ and from (1), (4), (5), that $Z_{x_{0}+h_{n}}\left(w_{0}\right)=f\left(x_{0}+h_{m}\right)$. Hence we deduce from (2) that $\left|f\left(x_{0}+h_{m}\right)-f\left(x_{0}+h_{n}\right)\right| \leqq \epsilon_{1}$, which contradicts (4) and shows that our supposition that (4) holds is never true. But the continuous function

$$
\left|f\left(x+h_{m}\right)-f\left(x+h_{n}\right)\right| \leqq \epsilon_{1}
$$

when $x=0$ and never lies in the range $\epsilon_{1}<\xi<\eta$; so it is always $\leqq \epsilon_{1}$. Hence $\lim _{k \rightarrow \infty} f\left(x+h_{k}\right)$ exists uniformly in $x$.

4. Proof of Finiteness of $P$. It will next be shown that $P$ is finite. By the definition of $P$, we know that there are $P$ numbers $z_{j}^{*},(j=1, \cdots, P)$, for which it is possible to find sequences $h_{1}{ }^{(j)}, h_{2}{ }^{(j)}, \cdots$ of $\mathbb{R}$ for which

$$
\lim _{k \rightarrow \infty} f\left(h_{k}^{(j)}\right)=z_{j}^{*}
$$

moreover, the $z_{j}^{*}$ are the only such numbers. If $P$ is infinite, the set of numbers $z_{j}^{*}$ must have a limit point, and we can choose $Q$ and $R$ such that the positive number $\phi=\left|z_{Q}{ }^{*}-z_{R}{ }^{*}\right|$ is less than or equal to half the number $\eta$ which occurs in the implicit function theorem. We next choose a positive number $\delta$ which does not exceed the number $\nu$ occurring in the implicit function theorem and such that when $|w| \leqq \delta$,

$$
\left|Z_{x}(w)-f(x)\right| \leqq \frac{\phi}{4}
$$

By the definition of $R$, we can choose an integer $m$ so that

$$
\left|f\left(h_{m}^{(Q)}\right)-z_{Q}^{*}\right| \leqq \frac{\phi}{4} \text { and }\left|f\left(h_{m}^{(R)}\right)-z_{R}^{*}\right| \leqq \frac{\phi}{4}
$$

and such that for all $x$ and all $z$ in 5,

$$
\begin{aligned}
& \left|F\left(x+h_{m}^{(Q)}, z\right)-F(x, z)\right| \leqq \frac{\delta}{2}, \\
& \left|F\left(x+h_{m}^{(R)}, z\right)-F(x, z)\right| \leqq \frac{\delta}{2} .
\end{aligned}
$$


Since $F\left[h_{m}{ }^{(Q)}, f\left(h_{m}^{(Q)}\right)\right]=0$ it follows from (9) that if

$$
w_{0}=F\left[h_{m}^{(R)}, f\left(h_{m}^{(Q)}\right)\right],
$$

then $\left|w_{0}\right| \leqq \delta \leqq \nu$.

Now $Z_{h_{m}^{(R)}}\left(w_{0}\right)$ is the one and only number which satisfies both $\left|Z_{h_{m}^{(R)}}\left(w_{0}\right)-f\left(h_{m}{ }^{(R)}\right)\right| \leqq \eta$, and $F\left[h_{m}{ }^{(R)}, \quad Z_{h_{m}^{(R)}}\left(w_{0}\right)\right]=w_{0}$. But $F\left[h_{m}{ }^{(R)}, f\left(h_{m}{ }^{(Q)}\right)\right]=w_{0}$, and from (8) and the definition of $\phi$, it can be shown that $\left|f\left(h_{m}{ }^{(Q)}\right)-f\left(h_{m}{ }^{(R)}\right)\right| \leqq(3 / 2) \phi<\eta$. Thus $Z_{h_{m}^{(R)}}\left(w_{0}\right)=f\left(h_{m}{ }^{(Q)}\right)$; and, from (7), $\left|f\left(h_{m}{ }^{(Q)}\right)-f\left(h_{m}{ }^{(R)}\right)\right| \leqq \phi / 4$. Hence, from (8), we obtain $\left|z_{Q}^{*}-z_{R}{ }^{*}\right| \leqq(3 / 4) \phi$, which contradicts the definition of $\phi$ and shows that $P$ is finite.

5. Uniformity of the Limits. In this section it will be shown that for any sequence $h_{1}, h_{2}, \cdots$ of $R$, we have uniformly in $x$

$$
\lim _{K \rightarrow \infty} f\left(x+P h_{K}\right)=f(x) .
$$

As before, let $\lim _{K \rightarrow \infty} f\left(h_{K}^{(j)}\right)=z_{j}^{*}$, where $z_{1}^{*}, \cdots, z_{P}{ }^{*}$ are the $P$ numbers that can be obtained in this way. Then since $h_{1}, h_{2}, \cdots$ is in $R$, there exist functions $z_{1}(x), \cdots, z_{P}(x)$ such that uniformly in $x, \lim _{K \rightarrow \infty} f\left(x+h_{K}^{(j)}\right)=z_{j}(x),(j=1, \cdots, P)$, where of course $z_{j}(0)=z_{j}^{*},(j=1, \cdots, P)$. Now let $k_{1}{ }^{(j)}, k_{2}{ }^{(j)}, \cdots$ be an element of $R$ such that $\lim _{K \rightarrow \infty} f\left(k_{K}^{(j)}\right)=z_{j}^{*}$ and let $l_{1}^{(j)}, l_{2}{ }^{(j)}, \cdots$ be the sequence $h_{1}{ }^{(j)}, k_{1}{ }^{(j)}, h_{2}^{(j)}, k_{2}{ }^{(j)}, \ldots$. Clearly $l_{1}^{(j)}, l_{2}^{(j)}, \ldots$ is an element of $R$, and hence uniformly in $x, \lim _{K \rightarrow \infty} f\left(x+k_{K}^{(j)}\right)=z_{j}(x)$. Thus the $P$ functions $z_{1}(x), \cdots, z_{P}(x)$ are the only functions $z(x)$ such that $\lim _{K \rightarrow \infty} f\left(x+k_{K}\right)=z(x)$ uniformly in $x$ for some sequence $k_{1}, k_{2}, \cdots$ of $R$.

Because of the uniformity of the limits, we see that the sum and difference of any two elements of $R$ belongs to $R$; and that uniformly in $x$

$$
\lim _{K \rightarrow \infty} z_{i}\left(x+h_{K}^{(j)}\right)=\lim _{K \rightarrow \infty} f\left(x+h_{K}^{(i)}+h_{K}^{(j)}\right), \quad(i=1, \cdots, P) .
$$

But the right member is one of the $z_{j}(t)$, and hence translating by $h_{K}{ }^{(j)}$ and taking the limit effects a permutation $S_{j}$ on the $P$ functions. Moreover, any other sequence $k_{1}, k_{2}, \cdots$ which takes $f(x)$ into $z_{j}(x)$ effects the same permutation $S_{j}$, and each sequence of $R$ effects one of the permutations $S_{1}, \cdots, S_{P}$. Finally, $h_{K}^{(i)}+h_{K}^{(j)}$ effects the permutation $S_{i} S_{j}=S_{j} S_{i}$; the sequence 
$0,0, \cdots$ effects the identity; and the sequence $-h_{K}^{(i)}$ effects the inverse of $S_{i}$; so these permutations form a group of order $P$. Hence the $P$ th power of each element is the identity, and (11) follows.

6. Completion of the Proof. The proof of the theorem will now be completed by considering any sequence $h_{1}, h_{2}, \ldots$ such that $\lim _{K \rightarrow \infty} F\left(x+h_{K}, z\right)=F(x, z)$ uniformly in $x$ and $z$ for all $x$ and all $z$ in $\sqrt{5}$. Suppose that it is not true that uniformly in $x$

$$
\lim _{K \rightarrow \infty} f\left(x+P h_{K}\right)=f(x) .
$$

Then there exist $\epsilon>0$ and an infinite sub-sequence $h_{1}{ }^{\prime}, h_{2}{ }^{\prime}, \cdots$ of $h_{1}, h_{2}, \cdots$ and a sequence of numbers $x_{1}, x_{2}, \cdots$ such that for all $K$,

$$
\left|f\left(x_{K}+P h_{K}^{\prime}\right)-f\left(x_{K}\right)\right|>\epsilon .
$$

Let $h_{1}{ }^{\prime}, h_{2}{ }^{\prime \prime}, \cdots$ be a sub-sequence of $h_{1}{ }^{\prime}, h_{2}{ }^{\prime}, \cdots$ such that $f\left(h_{1}^{\prime \prime}\right), f\left(h_{2}^{\prime \prime}\right), \cdots$ converges. Then ${h_{1}^{\prime \prime}}^{\prime \prime}, h_{2}{ }^{\prime \prime}, \cdots$ is an element of $R$, and we have, uniformly in $x, \lim _{K \rightarrow \infty} f\left(x+P h_{K}{ }^{\prime \prime}\right)=f(x)$. But this contradicts (13) and hence (12) holds uniformly in $x$. It follows that, uniformly in $x, \lim _{K \rightarrow \infty} f\left[P\left(x+h_{K}\right)\right]=f(P x)$; and hence that $f(P x)$ is a.p. Moreover, the module of $f(P x)$ is contained in the module of $F(x, z)$, and the theorem follows.

7. Algebraic Functions. Let $D\left(a_{1}, \cdots, a_{n}\right)$ denote the discriminant of $z^{n}+a_{1} z^{n-1}+\cdots+a_{n}=0$; let $\phi_{1}(x), \cdots, \phi_{n}(x)$ be u.a.p. functions such that $\left|D\left[\phi_{1}(x), \cdots, \phi_{n}(x)\right]\right|>\delta>0$ for all $x$; and let $F(x, z)=z^{n}+\phi_{1}(x) z^{n-1}+\cdots+\phi_{n}(x)$. If $f(x)$ is any one of the $n$ continuous solutions of

$$
F[x, f(x)]=0,
$$

then it is clear that $f(x)$ and $F(x, z)$ satisfy the conditions of our theorem, except possibly the condition that for all $x$, $\left|F_{z}[x, f(x)]\right|>\lambda>0$. That this condition also holds can be seen by considering the closures $\Re$ and $\&$ of the $n$ - and $(n+1)$-dimensional vectors $\left[\phi_{1}(x), \cdots, \phi_{n}(x)\right]$ and $\left[f(x), \phi_{1}(x), \cdots, \phi_{n}(x)\right]$. If $\left[b, a_{1}, \cdots, a_{n}\right]$ is in $\mathfrak{R}$,

$$
b^{n}+a_{1} b^{n-1}+\cdots+a_{n}=0,
$$

and $\left|D\left[a_{1}, \cdots, a_{n}\right]\right| \geqq \delta>0$, so that

$$
n b^{n-1}+(n-1) a_{1} b^{n-2}+\cdots+a_{n-1} \neq 0 \text {. }
$$


Since $\mathbb{R}$ is closed and bounded, our condition follows. We can now draw the conclusion of our theorem. In particular, since the limit of a sequence of $R$ is a solution of $F(0, z)=0$, we have $P \leqq n$. Thus we see that $f(x)$ is u.a.p. and its module is contained in the set of numbers obtained by dividing each element of the module of $\phi_{1}(x), \cdots, \phi_{n}(x)$ by $P$. This theorem was proved by Walther, ${ }^{*}$ except that his characterization of the module is less incisive in that he uses $n$ ! in place of $P \leqq n$.

8. Generalization. Theorem 1 may be generalized by allowing $z$ to be an $n$-dimensional vector $z_{1}, \cdots, z_{n}$ and $f(x)$ and $F(x, z)$ vector functions $f_{1}(x), \cdots, f_{n}(x)$ and $F_{1}(x, z), \cdots, F_{n}(x, z)$. The $f_{i}, F_{i}$, and $z_{i}$ are all real or all complex, while $x$ remains a single real variable. The distance between $z$ and $z^{\prime}$ is to be $\max _{i}\left|z_{i}-z_{i}^{\prime}\right|$, and similarly for $f$ and $F$. The derivative $\partial F / \partial z$ is to be replaced by all the partial derivatives $\partial F_{i} / \partial z_{j}$ in (c) and (e), and by

$$
\frac{\partial\left[F_{1}, \cdots, F_{n}\right]}{\partial\left[z_{1}, \cdots, z_{n}\right]}
$$

in (d). The condition (e) is to be strengthened by requiring uniform boundedness at the stipulated points as well as uniform continuity; and in view of this, the condition (b) may be omitted from the statement of the theorem. The original proof holds without change, and need not be repeated. If we omit the part of the theorem concerning the modules, we can also allow $x$ to become a point in a connected space $E$ as described by Bochner in his paper Abstracte fastperiodische Funktionen. $\dagger$

9. Stepanoff Functions. In the following theorem $n$-dimensional vectors will be used, and the symbol $\|P\|$ will denote the norm $\Sigma\left|p_{i}\right|$ of the vector $P: p_{1}, \cdots, p_{n}$. Vectors will be added, multiplied by scalars, differentiated, and integrated by having the operation performed on each component.

Theorem 2. Let $\Phi(z, P) \equiv \Phi\left(z, p_{1}, p_{2}, \cdots, p_{n}\right) \equiv z^{n}+p_{1} z^{n-1}$ $+\cdots+p_{n}$, and let $\Delta(P)$ be the discriminant of $\Phi(z, P)=0$. Let $F(t)$ be an S.a.p. vector function such that

* Algebraische Funktionen der fastperiodischen Funktionen, Monatshefte für Mathematik, vol. 40 (1933), pp. 444-457.

$\dagger$ Acta Mathematica, vol. 61, pp. 149-184, especially p. 150. 


$$
\phi(t, u)=\max _{0<y \leq u}\left\|F(t)-\frac{1}{y} \int_{t}^{t+y} F(\xi) d \xi\right\|
$$

converges in measure as $u \rightarrow 0$ uniformly on every interval of unit length; and let positive numbers $\lambda$ and $\delta_{1}$ exist such that

$$
\left|\Delta\left[\frac{1}{u} \int_{t}^{t+u} F(\xi) d \xi\right]\right| \geqq \lambda
$$

for all positive $u \leqq \delta_{1}$ and all $t$. Then the equation $\Phi[z(t), F(t)]=0$ has an S.a.p. solution.

It should be noted that the condition on $\phi(t, u)$ is a restriction only so far as the uniformity of the convergence is concerned. As $u \rightarrow 0, \phi(t, u)$ must automatically converge in measure on every bounded interval.

To establish this theorem, let $I$ be the interval $-\infty<t<+\infty$, $R_{0}$ the two-dimensional set $-\infty<t<+\infty, 0<u \leqq \delta$, and $R$ the set consisting of $R_{0}$ together with the points $t \subset I-E_{0}, u=0$, where $E_{0}$ is the set of measure zero on which it is not true that

$$
\lim _{u \rightarrow 0^{+}} \frac{1}{u} \int_{t}^{t+u} F(\xi) d \xi=\frac{d}{d t} \int_{0}^{t} F(\xi) d \xi=F(t) .
$$

Let

$$
\frac{1}{u} \int_{t}^{t+u} F(\xi) d \xi=F(t, u),
$$

and $F(t)=F(t, 0)$; then $F(t, u)$ is a continuous function of $u$ on $R$ and is continuous in $t$ and $u$ on $R_{0}$. Since $R_{0}$ is simply connected, it follows that if we choose a value for the solution of $\Phi[z(t, u), F(t, u)]$ at one point, say $t=0, u=\delta$, and require that $z(t, u)$ be continuous, then $z(t, u)$ is uniquely defined on our strip; and if we define $z(t, 0)$ as $\lim _{u \rightarrow 0^{+}} z(t, u)$, the function $z(t, u)$ is uniquely defined on $R$. Moreover $|\Delta[F(t, u)]| \geqq \lambda$ on $R$; it follows that if we change the value of $z(0, \delta)$ to another root of $\Phi\left[z, F\left(0, \delta_{1}\right)\right]=0$, the value of $z(t, u)$ will be changed at every point $R$. Thus the two values of $z(t, 0)$ will differ on a set of positive measure.

Suppose now that the value of $z(0, \delta)$ has been chosen and that $z(t, u)$ is accordingly defined on $R$. Let $z(t, 0)$ be defined 
arbitrarily on $E_{0}$ so as to satisfy $\Phi[z, F(t)]=0$. Then $z(t, 0)$ is a solution of this equation for all $t$. It remains to prove that this solution is S.a.p.

Let $\epsilon>0$, and let $L$ be an $(\epsilon / 9)$-inclusion interval of $F(t)$. Let $\eta \leqq \epsilon / 9$ be a positive number such that if $S$ is any measurable subset of the interval $0 \leqq t \leqq L+1$ whose measure does not exceed $\eta$, then $\int_{S}\|F(\xi)\| d \xi \leqq \epsilon / 9$. Let $N$ be so great that

$$
\sup _{t} m[\|F(\xi)\|>N]_{t}^{t+1} \leqq \frac{\eta}{2}
$$

Here $m[S]_{a}^{b}$ denotes the measure of the part of the set $S$ incluced in the interval $a \leqq \xi \leqq b$. Such a choice of $N$ is possible since $\int_{t}^{t+1}\|F(\xi)\| d \xi$ is bounded. Let $I_{t}$ denote the interval $t \leqq \xi \leqq t+1$, and let $E_{1}$ be the set of values of $t$ such that $\|F(t)\|$ $>N$. Then on $I-\left(E_{0}+E_{1}\right),|\Delta[F(t)]| \geqq \lambda$ and $F(t)$ is bounded; so by the implicit function theorem there exist positive numbers $h$ and $r$, such that whenever $\|P-F(t)\| \leqq r, t \subset I-\left(E_{0}+E_{1}\right)$, then there exists one and only one complex number $Z_{t}(P)$ which satisfies simultaneously $\Phi\left[Z_{t}(P), P\right]=0$ and $\left|Z_{t}(P)-z(t, 0)\right|$ $\leqq h$. Moreover, we can choose a positive number $\nu$ which does not exceed $r$ or $\epsilon / 9$ such that whenever $\|F(t)-P\| \leqq \nu, t \subset I$ $-\left(E_{0}+E_{1}\right)$, then $\left|Z_{t}(P)-z(t, 0)\right| \leqq \epsilon / 9$. Now choose $\bar{\delta}$ such that when $0 \leqq u \leqq \bar{\delta}$, then for all $t, m[\|\phi(\xi, u)\|>\nu]_{t}^{t+1} \leqq \eta / 2$; and such that on this range, $0 \leqq u \leqq \bar{\delta}$, we have for all $t$

$$
\int_{t}^{t+1}\|F(\xi)-F(\xi, u)\| d \xi \leqq \frac{\epsilon}{9}
$$

Finally, let $E_{2}$ be the set of values of $t$ at which $\|\phi(t, \bar{\delta})\|>\nu$. Then $\sup _{t} m\left[I_{t} E_{2}\right] \leqq \eta / 2$ and $\sup _{t} m\left[I_{t}\left(E_{0}+E_{1}+E_{2}\right)\right] \leqq \eta$.

Let $t$ be any real number and let $t$ equal $s+\tau$, where $\tau$ is an $(\epsilon / 9)$-translation number of $F(t)$ and $0 \leqq s \leqq L$. If $f_{1}(\xi, u), \cdots$, $f_{n}(\xi, u)$ are the components of $F(\xi, u)$, then it follows from $0=\Phi[z(\xi, u), F(\xi, u)]$ that whenever $|z(\xi, u)| \geqq 1,|z(\xi, u)|$ $=\mid-f_{1}(\xi, u)-f_{2}\left(\xi, u[z(\xi, u)]^{-1}-\ldots-f_{n}(\xi, u)[z(\xi, u)]^{1-n} \mid\right.$ $\leqq\|F(\xi, u)\|$. Thus for all $\xi, u$, we have $|z(\xi, u)|<1+\|F(\xi, u)\|$ $\leqq 1+\|F(\xi-\tau, 0)\|+\|F(\xi, 0)-F(\xi-\tau, 0)\|+\|F(\xi, u)-F(\xi, 0)\|$, and for $0 \leqq u \leqq \bar{\delta}$, 


$$
\begin{aligned}
& \int_{I_{t\left(E_{0}+E_{1}+E_{2}\right)}}|z(\xi, u)| d \xi \leqq m\left[I_{t}\left(E_{0}+E_{1}+E_{2}\right)\right] \\
+ & \int_{I_{t\left(E_{0}+E_{1}+E_{2}\right)}}\|F(\xi-\tau)\| d \xi+\int_{t}^{t+1}\|F(\xi)-F(\xi-\tau)\| d \xi \\
+ & \int_{t}^{t+1}\|F(\xi, u)-F(\xi)\| d \xi \leqq \eta+\frac{3 \epsilon}{9} \leqq \frac{4 \epsilon}{9}
\end{aligned}
$$

since

$$
\int_{I_{t\left(E_{0}+E_{1}+E_{2}\right)}}\|F(\xi-\tau)\| d \xi=\int_{S}\|F(\xi)\| d \xi,
$$

where $m(S) \leqq \eta$ and $S \subset I_{t-\tau}$ which is contained in the interval $0 \leqq \xi \leqq L+1$. Thus for $0 \leqq u \leqq \bar{\delta}$,

$$
\int_{I_{t}\left(E_{0}+E_{1}+E_{2}\right)}|z(\xi, 0)-z(\xi, u)| d \xi \leqq \frac{8 \epsilon}{9} .
$$

Now on $I-\left(E_{0}+E_{1}+E_{2}\right),\|\phi(\xi, \bar{\delta})\| \leqq \nu ;$ and whenever $\|P-F(\xi)\| \leqq \nu$, then $\left|Z_{\xi}(P)-z(\xi, 0)\right| \leqq \epsilon / 9$. Therefore we may write $\Phi\left\{Z_{\xi}[F(\xi, u)], F(\xi, u)\right\}=0, \Phi[z(\xi, u), F(\xi, u)]=0$, and $\left|Z_{\xi}[F(\xi, u)]-z(\xi, 0)\right| \leqq \epsilon / 9$ if $u \leqq \bar{\delta}$ and $\xi \subset I-\left(E_{0}+E_{1}+E_{2}\right)$. But for a fixed $\xi$ in $I-\left(E_{0}+E_{1}+E_{2}\right), z(\xi, u)-Z_{\xi}[F(\xi, u)]$ is continuous for $u \leqq \bar{\delta}$ and is zero when $u=0$. Thus by the uniqueness of the solution $Z_{\xi}(P)$ in its neighborhood, we have $z(\xi, u)=Z_{\xi}[F(\xi, u)]$, and hence $|z(\xi, u)-z(\xi, 0)| \leqq \epsilon / 9$ when $0 \leqq u \leqq \bar{\delta}$ and $\xi \subset I-\left(E_{0}+E_{1}+E_{2}\right)$. Hence for $0 \leqq u \leqq \bar{\delta}$,

$$
\int_{I_{t-\left(E_{0}+E_{1}+E_{2}\right)}}|z(\xi, 0)-z(\xi, u)| d \xi \leqq \int_{I_{t}} \frac{\epsilon}{9} d \xi \leqq \frac{\epsilon}{9},
$$

and $\int_{I_{t}}|z(\xi, 0)-z(\xi, u)| d \xi \leqq \epsilon$. This inequality does not depend on $t$ and we have that $z(\xi, 0)$ is the Stepanoff limit of $z(\xi, u)$, and hence is S.a.p.

CoROLlary. Under the conditions of the theorem, the equation $\Phi[z(t), F(t)]=0$ will always have an infinite number of essentially distinct S.a.p. solutions, provided $n>1$.

For it is clear from the argument used in the theorem that $\Phi[z(t), F(t)]=0$ has at least $n$ essentially distinct solutions $z_{1}(t), \cdots, z_{n}(t)$, no two of which are equal on a set of positive 
measure. Let $L$ be a positive number and let $z(t, L)$ be equal either to $z_{1}(t)$ or $z_{2}(t)$ according as $[t / L]$ is even or odd. It is clear that $z(t, L)$ is an S.a.p. solution of $\Phi[z(t), F(t)]=0$ for each positive $L$, and that all these solutions are essentially distinct. Of course many other solutions could be constructed in a similar way.

Brown University and Princeton University

\section{ON A LEMMA OF FEJÉR*}

BY LINCOLN LA PAZ

1. Simple Integrals. In an important paper L. Fejér † has verified and used the following lemma.

Lemma A. If for a problem of minimizing an integral

$$
I_{1}=\int_{x_{1}}^{x_{2}} \phi\left(y^{\prime}\right) \cdot f(x, y) d x,
$$

the Euler equation in normal form is

$$
y^{\prime \prime}=F\left(x, y, y^{\prime}\right),
$$

then for a problem of minimizing the integral

$$
J_{1}=\int_{x_{1}}^{x_{2}}\left[\phi\left(y^{\prime}\right) / f(x, y)\right] d x,
$$

the Euler equation in normal form is

$$
y^{\prime \prime}=-F\left(x, y, y^{\prime}\right) \text {. }
$$

The following generalization of Fejér's lemma is proved in this note.

* Presented to the Society, December 2, 1933.

$\dagger$ L. Fejér, Das Ostwaldsche Prinzip in der Mechanik, Mathematische Annalen, vol. 61 (1905), p. 432.

$\ddagger$ In everything that follows, the range of the indices $i, j, k, \mu, \nu$ is from 1 to $n$ and $\mu$ and $\nu$ are umbral. 